\title{
ON THE ASYMMETRIC TELEGRAPH PROCESSES
}

\author{
OSCAR LÓPEZ*** AND \\ NIKITA RATANOV, ${ }^{* * * *}$ Universidad del Rosario
}

\begin{abstract}
We study the one-dimensional random motion $X=X(t), t \geq 0$, which takes two different velocities with two different alternating intensities. The closed-form formulae for the density functions of $X$ and for the moments of any order, as well as the distributions of the first passage times, are obtained. The limit behaviour of the moments is analysed under nonstandard Kac's scaling.
\end{abstract}

Keywords: Asymmetric telegraph process; first passage time; Kac's asymptotics; moments; modified Bessel function; Kummer function

2010 Mathematics Subject Classification: Primary 60J27

Secondary 60J65; 60F05; 60K99

\section{Introduction}

The main subject of study in this paper is the mathematical description of noninteracting particles moving in one dimension with alternating finite velocities; this is the so-called telegraph (or telegrapher's) stochastic process. Beginning with the 1956 lecture notes by Kac [13], the telegraph processes and their numerous generalisations have been studied in great detail; see, e.g. [2], [5], [4], [7], [17], [18], [19], [20], [24], with applications in physics [23], biology [10], [11], ecology [16], and, more recently, in financial market modelling [21] (see also the bibliographies in these papers).

In the symmetric case, where the particles move with symmetric velocities $\pm c$ and switch their directions with the intensity $\lambda, \lambda>0$, the density function $p=p(x, t)$ of particles' positions satisfies the hyperbolic second-order differential equation

$$
\frac{\partial^{2} p(x, t)}{\partial t^{2}}+2 \lambda \frac{\partial p(x, t)}{\partial t}=c^{2} \frac{\partial^{2} p(x, t)}{\partial x^{2}}, \quad t>0,
$$

known as the telegraph (or damped wave) equation; see [13]. Under a scaling condition on $\lambda$ and $c$ such that $c^{2} / \lambda \rightarrow \sigma^{2}$ as $\lambda \rightarrow \infty$, it was noticed by Kac that (1.1) converges to the heat equation and that the dynamical particles' distribution weakly converges to the diffusion with the diffusion coefficient $\sigma^{2}$.

If the velocities are different in different directions, the particles' movement holds only an additional drift. In this paper we assume that the movement is asymmetric not only with respect to the velocities but also with respect to the distinct switching intensities. This type of asymmetry of the movement is necessary in order to develop adequate applications of the theory in science and technology, as well as in financial modelling. In the latter case the asymmetry serves as a natural medium for the description of a risk-neutral measure; see [21].

Received 25 January 2013; revision received 14 June 2013.

* Postal address: Universidad del Rosario, Cl. 12c, No. 4-69, Bogotá, Colombia.

** Email address: oscar.lopez@urosario.edu.co

*** Email address: nratanov@urosario.edu.co 
In comparison with the classical case, the properties of the asymmetric telegraph processes are less well known; see [2], [21], and, with symmetric switching intensities, [22]. Moreover, some existing results are formulated with mistakes.

In order to describe the distribution of the telegraph process, we systematically exploit a noninfinitesimal approach based on integral equations instead of the traditional differential telegraph equation (1.1). We also derive moment generating functions and conditional densities, given the initial direction and the number of switchings.

In the same manner we study some important functionals of the asymmetric telegraph processes. We obtain explicit formulae for the distributions of first passage times and for the moments. The distributions of the first passage times are described by using the Laplace transforms and their inversions.

The moments of the telegraph processes in the homogeneous symmetric setting have been described by Iacus and Yoshida, [12], in terms of modified Bessel functions; see also [14]. Our approach produces expressions that are a bit more simple. In this paper we present a survey of the explicit formulae of the moments in the asymmetric case by using confluent hypergeometric functions.

We also analyse the limit behaviour of the moments under Kac's scaling, when switching intensities are 'asymptotically' similar. Surprisingly, the standard result under Kac's condition does not hold without some additional assumptions on the rate of this similarity. Moreover, a very moderate difference in the asymptotic behaviour of switching intensities provides an additional drift in the limiting diffusion. This result is in accordance with the authors' previous study of the asymptotical distributions of the inhomogeneous jump-telegraph processes [15].

The paper is organised as follows. Section 2 deals with the detailed description of the distributions. We begin this section with the transition densities of telegraph processes, and then we present the explicit formulae for the moment generating functions and for the conditional distributions. Section 3 concerns the distributions of time $T(x)$ when the particle hits the fixed level $x$. In Section 4 we derive the formulae for the moments by using a similar technique in terms of linear combination of Kummer functions. The asymptotical behaviour of the moments under Kac's condition is analysed in Section 5.

\section{Asymmetric telegraph process and its transition densities}

Let $\left(\Omega, \mathfrak{F},\left\{\mathfrak{F}_{t}\right\}_{t \geq 0}, \mathbb{P}\right)$ be a filtered probability space. First, we consider a two-state Markov process $\varepsilon=\varepsilon(t), \varepsilon(t) \in\{0,1\}, t \geq 0$, with alternating transition intensities $\lambda_{0}>0$ and $\lambda_{1}>0$, such that the generator is defined by the matrix

$$
\boldsymbol{\Lambda}:=\left(\begin{array}{cc}
-\lambda_{0} & \lambda_{0} \\
\lambda_{1} & -\lambda_{1}
\end{array}\right) .
$$

The process $\varepsilon=\varepsilon(t), t \geq 0$, is assumed to be adapted to the filtration $\left\{\mathfrak{F}_{t}\right\}_{t \geq 0}$.

By definition, the point process of switching times $0=\tau_{0}<\tau_{1}<\tau_{2}<\cdots$ of the Markov process $\varepsilon$ has exponentially distributed and independent increments: for $n=0,1,2, \ldots$, $\mathbb{P}\left\{\tau_{n+1}-\tau_{n}>t \mid \mathfrak{F}_{\tau_{n}}\right\}=\exp \left(-\lambda_{\varepsilon\left(\tau_{n}\right)} t\right)$.

Let $c_{0}, c_{1} \in(-\infty, \infty)$ be two arbitrary real numbers, $c_{0}>c_{1}$. Consider the processes $V(t)=c_{\varepsilon(t)}$ and $X(t)=\int_{0}^{t} V(s) \mathrm{d} s, t \geq 0$. The process $X=X(t), t \geq 0$, is called the inhomogeneous (or asymmetric) telegraph process with the alternating states $\left(c_{0}, \lambda_{0}\right)$ and $\left(c_{1}, \lambda_{1}\right)$. Note that process $X$ starts at the origin, $X(0)=0$.

We fix the initial state $\varepsilon(0)=i \in\{0,1\}$. Hence, the process $X$ starts with the fixed velocity $c_{i}$; at time $\tau_{1}=\tau_{1}^{(i)}$ (which is exponentially distributed with the parameter $\lambda_{i}$ ) X(t) takes the 
opposite state $1-i$ with the velocity $c_{1-i}$ and the switching intensity $\lambda_{1-i}$. Thus, for any $t>0$, we have the equality in distribution

$$
X(t) \stackrel{\mathrm{D}}{=} c_{i} t \mathbf{1}_{\left\{t<\tau_{1}\right\}}+\left[c_{i} \tau_{1}+\tilde{X}\left(t-\tau_{1}\right)\right] \mathbf{1}_{\left\{t>\tau_{1}\right\}} .
$$

Here the process $\tilde{X}=\tilde{X}(t)$ is a telegraph process independent of $X$, driven by the same parameters, but $\tilde{X}$ starts from the opposite state $1-i$.

Throughout the paper, we use the notation

$$
2 c=c_{0}-c_{1}, \quad 2 a=c_{0}+c_{1}, \quad 2 \beta=\lambda_{0}-\lambda_{1}, \quad 2 \lambda=\lambda_{0}+\lambda_{1} .
$$

\subsection{Transition densities}

Denote by $\mathbb{P}_{i}\{\cdot\}=\mathbb{P}\{\cdot \mid \varepsilon(0)=i\}$ and $\mathbb{E}_{i}\{\cdot\}=\mathbb{E}\{\cdot \mid \varepsilon(0)=i\}$ the conditional probabilities and the conditional expectations respectively, given the initial state value $\varepsilon(0)=i$ for $i=0,1$. Denote by $p_{i}(x, t), i=0,1$, the density functions of $X(t), t \geq 0: p_{i}(x, t):=$ $\mathbb{P}_{i}\{X(t) \in \mathrm{d} x\} / \mathrm{d} x, i=0,1$, which can be decomposed as

$$
p_{i}(x, t)=\sum_{n=0}^{\infty} p_{i}(x, t ; n) .
$$

Here

$$
p_{i}(x, t ; n):=\mathbb{P}_{i}\{X(t) \in \mathrm{d} x, N(t)=n\} / \mathrm{d} x,
$$

where $N(t), t \geq 0$, is the inhomogeneous counting Poisson process related to the rates $\lambda_{0}$ and $\lambda_{1}$.

Applying (2.2), we immediately get the following two sets of integral equations:

$$
\begin{aligned}
& p_{0}(x, t)=\mathrm{e}^{-\lambda_{0} t} \delta\left(x-c_{0} t\right)+\int_{0}^{t} p_{1}\left(x-c_{0} \tau, t-\tau\right) \lambda_{0} \mathrm{e}^{-\lambda_{0} \tau} \mathrm{d} \tau, \\
& p_{1}(x, t)=\mathrm{e}^{-\lambda_{1} t} \delta\left(x-c_{1} t\right)+\int_{0}^{t} p_{0}\left(x-c_{1} \tau, t-\tau\right) \lambda_{1} \mathrm{e}^{-\lambda_{1} \tau} \mathrm{d} \tau,
\end{aligned}
$$

and

$$
\begin{aligned}
& p_{0}(x, t ; 0)=\mathrm{e}^{-\lambda_{0} t} \delta\left(x-c_{0} t\right), \quad p_{1}(x, t ; 0)=\mathrm{e}^{-\lambda_{1} t} \delta\left(x-c_{1} t\right), \\
& p_{0}(x, t ; n)=\int_{0}^{t} p_{1}\left(x-c_{0} \tau, t-\tau ; n-1\right) \lambda_{0} \mathrm{e}^{-\lambda_{0} \tau} \mathrm{d} \tau, \quad n \geq 1, \\
& p_{1}(x, t ; n)=\int_{0}^{t} p_{0}\left(x-c_{1} \tau, t-\tau ; n-1\right) \lambda_{1} \mathrm{e}^{-\lambda_{1} \tau} \mathrm{d} \tau, \quad n \geq 1,
\end{aligned}
$$

where $\delta$ is the Dirac $\delta$-function and the term $\mathrm{e}^{-\lambda_{i} t} \delta\left(x-c_{i} t\right)$ corresponds to the movement without any change of the initial direction. Here, and everywhere below, we set $\int_{-\infty}^{\infty} \delta(x-c \tau) \varphi(\tau) \mathrm{d} \tau=$ $\varphi(x / c) / c$ for any continuous test function $\varphi$.

Differentiating the integral equations of (2.5) and then integrating the result by parts, we easily derive the equivalent Cauchy problem

$$
\begin{aligned}
\frac{\partial p_{0}}{\partial t}(x, t)+c_{0} \frac{\partial p_{0}}{\partial x}(x, t) & =-\lambda_{0} p_{0}(x, t)+\lambda_{0} p_{1}(x, t), \\
\frac{\partial p_{1}}{\partial t}(x, t)+c_{1} \frac{\partial p_{1}}{\partial x}(x, t) & =-\lambda_{1} p_{1}(x, t)+\lambda_{1} p_{0}(x, t),
\end{aligned}
$$

for $t>0$, with the initial conditions $p_{0}(x, 0)=p_{1}(x, 0)=\delta(x)$. Indeed, applying the operator 
$\partial / \partial t+c_{0} \partial / \partial x$ to the first equation of (2.5) we have

$$
\begin{aligned}
\left(\frac{\partial}{\partial t}+c_{0} \frac{\partial}{\partial x}\right) p_{0}(x, t)= & -\lambda_{0} \mathrm{e}^{-\lambda_{0} t} \delta\left(x-c_{0} t\right)+p_{1}\left(x-c_{0} t, 0\right) \lambda_{0} \mathrm{e}^{-\lambda_{0} t} \\
& -\int_{0}^{t} \frac{\partial}{\partial \tau}\left[p_{1}\left(x-c_{0} \tau, t-\tau\right)\right] \lambda_{0} \mathrm{e}^{-\lambda_{0} \tau} \mathrm{d} \tau \\
= & -\lambda_{0} \mathrm{e}^{-\lambda_{0} t} \delta\left(x-c_{0} t\right)+p_{1}\left(x-c_{0} t, 0\right) \lambda_{0} \mathrm{e}^{-\lambda_{0} t} \\
& -p_{1}\left(x-c_{0} t, 0\right) \lambda_{0} \mathrm{e}^{-\lambda_{0} t}+\lambda_{0} p_{1}(x, t) \\
& -\lambda_{0} \int_{0}^{t} p_{1}\left(x-c_{0} \tau, t-\tau\right) \lambda_{0} \mathrm{e}^{-\lambda_{0} \tau} \mathrm{d} \tau \\
= & -\lambda_{0} p_{0}(x, t)+\lambda_{0} p_{1}(x, t)
\end{aligned}
$$

which produces (2.7a). The first equality here follows from

$$
\begin{gathered}
\left(\frac{\partial}{\partial t}+c_{0} \frac{\partial}{\partial x}\right) \delta\left(x-c_{0} t\right)=0 \\
\left(\frac{\partial}{\partial t}+c_{0} \frac{\partial}{\partial x}\right) p_{1}\left(x-c_{0} \tau, t-\tau\right)=-\frac{\partial}{\partial \tau}\left[p_{1}\left(x-c_{0} \tau, t-\tau\right)\right]
\end{gathered}
$$

and the second equality, by integration by parts. Equation (2.7b) is derived similarly.

We express the solutions of (2.5) (and (2.7)) by means of the variables

$$
\xi=\xi(x, t):=\frac{x-c_{1} t}{c_{0}-c_{1}} \quad \text { and } \quad t-\xi=t-\xi(x, t)=\frac{c_{0} t-x}{c_{0}-c_{1}} .
$$

These expressions (see below Proposition 2.1) repeat the existing results from [21], but we include them for completeness of presentation.

Note that $0<\xi(x, t)<t$, if $x \in\left(c_{1} t, c_{0} t\right)$. Using this notation, we define the functions $q_{i}(x, t ; n), i=0,1$. For $c_{1} t<x<c_{0} t$,

$$
q_{0}(x, t ; 2 n)=\frac{\lambda_{0}^{n} \lambda_{1}^{n}}{(n-1) ! n !} \xi^{n}(t-\xi)^{n-1}, \quad q_{1}(x, t ; 2 n)=\frac{\lambda_{0}^{n} \lambda_{1}^{n}}{(n-1) ! n !} \xi^{n-1}(t-\xi)^{n}
$$

for $n \geq 1$, and

$$
q_{0}(x, t ; 2 n+1)=\frac{\lambda_{0}^{n+1} \lambda_{1}^{n}}{(n !)^{2}} \xi^{n}(t-\xi)^{n}, \quad q_{1}(x, t ; 2 n+1)=\frac{\lambda_{0}^{n} \lambda_{1}^{n+1}}{(n !)^{2}} \xi^{n}(t-\xi)^{n}
$$

for $n \geq 0$. Let

$$
\theta(x, t)=\frac{1}{c_{0}-c_{1}} \mathrm{e}^{-\lambda_{0} \xi-\lambda_{1}(t-\xi)} \mathbf{1}_{\{0<\xi<t\}} .
$$

Proposition 2.1. The set of integral equations (2.6) has the following solution:

$$
p_{i}(x, t ; n)=q_{i}(x, t ; n) \theta(x, t), \quad n \geq 1, i=0,1 .
$$

Proof. Let $n \geq 2$. We substitute (2.12) and (2.9)-(2.11) directly into (2.6).

Note that, for $0 \leq \tau \leq t$ and $c_{1} t<x<c_{0} t$, the following identities hold:

$$
\xi\left(x-c_{0} \tau, t-\tau\right) \equiv \xi(x, t)-\tau, \quad \xi\left(x-c_{1} \tau, t-\tau\right) \equiv \xi(x, t) ;
$$


hence,

$$
\lambda_{i} \tau+\lambda_{0} \xi\left(x-c_{i} \tau, t-\tau\right)+\lambda_{1}\left(t-\tau-\xi\left(x-c_{i} \tau, t-\tau\right)\right) \equiv \lambda_{0} \xi(x, t)+\lambda_{1}(t-\xi(x, t)),
$$

for $i=0,1$. The latter equality means that

$$
\begin{gathered}
\mathrm{e}^{-\lambda_{0} \tau} \theta\left(x-c_{0} \tau, t-\tau\right) \equiv \theta(x, t) \mathbf{1}_{\{\tau<\xi(x, t)\}}, \\
\mathrm{e}^{-\lambda_{1} \tau} \theta\left(x-c_{1} \tau, t-\tau\right) \equiv \theta(x, t) \mathbf{1}_{\{\tau<t-\xi(x, t)\}} .
\end{gathered}
$$

To solve (2.6), first note that, owing to (2.13) and (2.14), the following identities are fulfilled: for $m, k=0,1,2, \ldots$,

$$
\begin{aligned}
\int_{0}^{t} \xi & \left(x-c_{0} \tau, t-\tau\right)^{m}\left(t-\tau-\xi\left(x-c_{0} \tau, t-\tau\right)\right)^{k} \mathrm{e}^{-\lambda_{0} \tau} \theta\left(x-c_{0} \tau, t-\tau\right) \mathrm{d} \tau \\
& =\theta(x, t)(t-\xi(x, t))^{k} \int_{0}^{\xi(x, t)}(\xi(x, t)-\tau)^{m} \mathrm{~d} \tau \\
& =\theta(x, t)(t-\xi(x, t))^{k} \frac{\xi(x, t)^{m+1}}{m+1}
\end{aligned}
$$

and

$$
\begin{aligned}
\int_{0}^{t} \xi\left(x-c_{1} \tau, t-\tau\right)^{m}\left(t-\tau-\xi\left(x-c_{1} \tau, t-\tau\right)\right)^{k} \mathrm{e}^{-\lambda_{1} \tau} \theta\left(x-c_{1} \tau, t-\tau\right) \mathrm{d} \tau \\
\quad=\theta(x, t) \xi(x, t)^{m} \int_{0}^{t-\xi(x, t)}(t-\tau-\xi(x, t))^{k} \mathrm{~d} \tau \\
\quad=\theta(x, t) \xi(x, t)^{m} \frac{(t-\xi(x, t))^{k+1}}{k+1} .
\end{aligned}
$$

With this to hand, we can easily see that the functions $p_{i}(x, t ; n), i=0,1$, which are defined by (2.9)-(2.12), satisfy the integral equations in (2.6).

For $n=1$, (2.6) can be solved by inserting the initial functions into the equations. Thus, from $p_{i}(x, t ; 0)=\mathrm{e}^{-\lambda_{i} t} \delta\left(x-c_{i} t\right)$ we obtain $p_{i}(x, t ; 1)=\lambda_{i} \theta(x, t)$ (see (2.10)-(2.12)).

By means of (2.4), summing up the results presented in (2.9)-(2.12) we obtain the solution to the equations in (2.5) (as well as in (2.7)): for $i=0,1$,

$$
\begin{aligned}
p_{i}(x, t)=\mathrm{e}^{-\lambda_{i} t} \delta\left(x-c_{i} t\right)+ & {\left[\lambda_{i} I_{0}\left(2 \sqrt{\lambda_{0} \lambda_{1} \xi(t-\xi)}\right)\right.} \\
& \left.+\sqrt{\lambda_{0} \lambda_{1}}\left(\frac{\xi}{t-\xi}\right)^{\frac{1}{2}-i} I_{1}\left(2 \sqrt{\lambda_{0} \lambda_{1} \xi(t-\xi)}\right)\right] \theta(x, t),
\end{aligned}
$$

where $I_{0}$ and $I_{1}$ denote the modified Bessel functions, i.e. $I_{0}(z)=\sum_{n=0}^{\infty}(z / 2)^{2 n} /(n !)^{2}$ and $I_{1}(z)=I_{0}^{\prime}(z)$.

\subsection{Moment generating functions and conditional distributions}

Consider, for arbitrary $z \in(-\infty, \infty)$,

$$
\psi_{i}(z, t ; n)=\mathbb{E}_{i}\left\{\mathrm{e}^{-z X(t)} \mathbf{1}_{\{N(t)=n\}}\right\}=\int_{-\infty}^{\infty} \mathrm{e}^{-z x} p_{i}(x, t ; n) \mathrm{d} x, \quad n \geq 1 .
$$


Note that $\psi_{i}(z, t ; 0)=\exp \left\{-t\left(\lambda_{i}+c_{i} z\right)\right\}, i=0,1$. The explicit formulae for $\psi_{i}(z, t ; n)$, $n \geq 1$, are given by Theorem 2.1 below.

Let $\Phi=\Phi(a, b ; z)={ }_{1} F_{1}(a, b ; z)$ be the confluent hypergeometric Kummer function,

$$
\Phi(a, b ; z)=\sum_{k=0}^{\infty} \frac{z^{k}(a)_{k}}{k !(b)_{k}},
$$

where $(a)_{k}=a(a+1)(a+2) \cdots(a+k-1)$ and $(a)_{0}=1$ (see [1, Chapter 13]).

Theorem 2.1. For any $t>0$ and integer $n \geq 1$, the functions $\psi_{i}, i=0,1$, have the form

$$
\begin{gathered}
\psi_{0}(z, t ; 2 n)=\lambda_{0}^{n} \lambda_{1}^{n} \frac{t^{2 n} \Phi(n, 2 n+1 ; 2(\beta+c z) t)}{(2 n) !} \exp \left\{-\left(\lambda_{0}+c_{0} z\right) t\right\}, \\
\psi_{1}(z, t ; 2 n)=\lambda_{0}^{n} \lambda_{1}^{n} \frac{t^{2 n} \Phi(n, 2 n+1 ;-2(\beta+c z) t)}{(2 n) !} \exp \left\{-\left(\lambda_{1}+c_{1} z\right) t\right\}, \\
\psi_{0}(z, t ; 2 n-1)=\lambda_{0}^{n} \lambda_{1}^{n-1} \frac{t^{2 n-1} \Phi(n, 2 n ; 2(\beta+c z) t)}{(2 n-1) !} \exp \left\{-\left(\lambda_{0}+c_{0} z\right) t\right\}, \\
\psi_{1}(z, t ; 2 n-1)=\lambda_{0}^{n-1} \lambda_{1}^{n} \frac{t^{2 n-1} \Phi(n, 2 n ;-2(\beta+c z) t)}{(2 n-1) !} \exp \left\{-\left(\lambda_{1}+c_{1} z\right) t\right\} .
\end{gathered}
$$

Here $2 \beta=\lambda_{0}-\lambda_{1}$ and $2 c=c_{0}-c_{1}$ (see the notation given in (2.3)).

Proof. We express the integrals in (2.15) by means of (2.9)-(2.12). Define

$$
I_{m, k}(z, t)=\int_{-\infty}^{\infty} \mathrm{e}^{-z x} \xi(x, t)^{m}(t-\xi(x, t))^{k} \theta(x, t) \mathrm{d} x, \quad m, k \geq 0,
$$

where $($ see $(2.8)) \xi(x, t)=\left(x-c_{1} t\right) /(2 c), t-\xi(x, t)=\left(c_{0} t-x\right) /(2 c)$, and $\theta=\theta(x, t)$ is defined by (2.11). After the substitution $x=c_{1} t+2 c \xi$, we have

$$
I_{m, k}(z, t)=\mathrm{e}^{-\left(c_{1} z+\lambda_{1}\right) t} \int_{0}^{t} \mathrm{e}^{-2(c z+\beta) \xi} \xi^{m}(t-\xi)^{k} \mathrm{~d} \xi
$$

Hence, by the integral representation of the confluent hypergeometric function (see 13.2.1 of [1])

$$
\frac{\Gamma(b-a) \Gamma(a)}{\Gamma(b)} \Phi(a, b, z)=\int_{0}^{1} \mathrm{e}^{z \xi} \xi^{a-1}(1-\xi)^{b-a-1} \mathrm{~d} \xi
$$

the function $I_{m, k}(z, t)$ is given by

$$
I_{m, k}(z, t)=t^{m+k+1} \mathrm{e}^{-\left(c_{1} z+\lambda_{1}\right) t} \frac{m ! k !}{(m+k+1) !} \Phi(m+1, m+k+2 ;-2(c z+\beta) t) .
$$

From the equality $\Phi(a, b, z)=\mathrm{e}^{z} \Phi(b-a, b,-z)$ (see 13.1.27 of [1]) we obtain

$$
I_{m, k}(z, t)=t^{m+k+1} \mathrm{e}^{-\left(c_{0} z+\lambda_{0}\right) t} \frac{m ! k !}{(m+k+1) !} \Phi(k+1, m+k+2 ; 2(c z+\beta) t) .
$$

With this to hand, by applying the explicit formulae (2.9)-(2.12) to (2.15) we derive (2.17)(2.20). This completes the proof. 
The moment generating functions $\psi_{i}(z, t)=\mathbb{E}_{i}\left\{\mathrm{e}^{-z X(t)}\right\}$ for $i=0,1$, can be obtained by summing up (2.17)-(2.20). We have the following result.

Corollary 2.1. Let $\psi_{i}(z, t)=\mathbb{E}_{i}\left\{\mathrm{e}^{-z X(t)}\right\}, i=0,1$. Then

$$
\begin{gathered}
\psi_{0}(z, t)=\mathrm{e}^{-\left(\lambda_{0}+c_{0} z\right) t}\left[1+\Psi_{0}(2(\beta+c z) t, t)+\lambda_{0} \Psi_{1}(2(\beta+c z) t, t)\right], \\
\psi_{1}(z, t)=\mathrm{e}^{-\left(\lambda_{1}+c_{1} z\right) t}\left[1+\Psi_{0}(-2(\beta+c z) t, t)+\lambda_{1} \Psi_{1}(-2(\beta+c z) t, t)\right] .
\end{gathered}
$$

Here

$$
\Psi_{0}(u, t)=\sum_{n=1}^{\infty} \frac{\lambda_{0}^{n} \lambda_{1}^{n} t^{2 n}}{(2 n) !} \Phi(n, 2 n+1 ; u), \quad \Psi_{1}(u, t)=\sum_{n=1}^{\infty} \frac{\lambda_{0}^{n-1} \lambda_{1}^{n-1} t^{2 n-1}}{(2 n-1) !} \Phi(n, 2 n ; u) .
$$

The noninfinitesimal approach allows us to simplify some known formulae (compare the expression for the characteristic function from [2] (see (5.3) of [2]) with the detailed formulae of (2.21) and with Remark 2.1 of the present paper).

Remark 2.1. Comparing (2.21) with (3.3) of [15], we can simplify the latter formulae, i.e.

$$
\begin{aligned}
& \psi_{0}(z, t)=\mathrm{e}^{-t(\lambda+a z)}\left(\cosh (t D)+(\lambda-c z) \frac{\sinh (t D)}{D}\right), \\
& \psi_{1}(z, t)=\mathrm{e}^{-t(\lambda+a z)}\left(\cosh (t D)+(\lambda+c z) \frac{\sinh (t D)}{D}\right),
\end{aligned}
$$

where $D=\sqrt{(\beta+c z)^{2}+\lambda_{0} \lambda_{1}}$ (here we use the notation given in $((2.3))$. See also Formula (5.3) of [2] for the characteristic function.

Finally, we consider the conditional densities

$$
f_{i}(x, t ; n):=\mathbb{P}_{i}\{X(t) \in \mathrm{d} x \mid N(t)=n\} / \mathrm{d} x, \quad n \geq 0, i=0,1 .
$$

In the symmetric case, where $\lambda_{0}=\lambda_{1}=\lambda$, it is well known (see, e.g. [6]) that, if $N(t)=n$, the times $\left\{\tau_{k}\right\}_{k=1}^{n}$ can be viewed as the order statistics from the uniform distribution in $[0, t]$. In this symmetric case $\mathbb{P}\{N(t)=n\}=\mathrm{e}^{-\lambda t}(\lambda t)^{n} / n$ ! and the conditional densities take the form

$$
f_{i}^{\mathrm{sym}}(x, t ; n)=\frac{n !}{\left(c_{0}-c_{1}\right)(\lambda t)^{n}} q_{i}(x, t ; n) \mathbf{1}_{\left\{c_{1} t<x<c_{0} t\right\}}, \quad i=0,1,
$$

where the $q_{i}$ are defined by (2.9)-(2.10) with $\lambda_{0}=\lambda_{1}=\lambda$. It is easy to see that the functions $f_{i}^{\mathrm{sym}}(x, t ; n), i=0,1$, do not depend on $\lambda$.

In the asymmetric case, where $\lambda_{0} \neq \lambda_{1}$, it is not so simple. Nevertheless, by definition, we have

$$
\begin{aligned}
f_{i}(x, t ; n) & =\frac{p_{i}(x, t ; n)}{\mathbb{P}_{i}\{N(t)=n\}} \\
& =\frac{p_{i}(x, t ; n)}{\int_{-\infty}^{\infty} p_{i}(y, t ; n) \mathrm{d} y} \\
& =\frac{p_{i}(x, t ; n)}{\psi_{i}(0, t ; n)}, \quad i=0,1,
\end{aligned}
$$


where the functions $p_{i}$ and $\psi_{i}$ are defined by (2.9)-(2.12) and (2.17)-(2.20) respectively. Therefore, for $n \geq 1$,

$$
\begin{gathered}
f_{0}(x, t ; 2 n)=\frac{(2 n) !}{2 c t^{2 n}} \frac{\xi^{n}(t-\xi)^{n-1}}{n !(n-1) !} \frac{\mathrm{e}^{-2 \beta(t-\xi)}}{\Phi(n, 2 n+1 ; 2 \beta t)} \\
f_{1}(x, t ; 2 n)=\frac{(2 n) !}{2 c t^{2 n}} \frac{\xi^{n-1}(t-\xi)^{n}}{(n-1) ! n !} \frac{\mathrm{e}^{-2 \beta \xi}}{\Phi(n, 2 n+1 ;-2 \beta t)} \\
f_{0}(x, t ; 2 n-1)=\frac{(2 n-1) !}{2 c t^{2 n-1}} \frac{\xi^{n}(t-\xi)^{n}}{(n !)^{2}} \frac{\mathrm{e}^{-2 \beta(t-\xi)}}{\Phi(n, 2 n ; 2 \beta t)} \\
f_{1}(x, t ; 2 n-1)=\frac{(2 n-1) !}{2 c t^{2 n-1}} \frac{\xi^{n}(t-\xi)^{n}}{(n !)^{2}} \frac{\mathrm{e}^{-2 \beta \xi}}{\Phi(n, 2 n ;-2 \beta t)}
\end{gathered}
$$

Here $\xi=\xi(x, t)$ is defined by (2.8).

\section{First passage time}

In this section we study the distribution of the first passage time

$$
T(x)=\inf \{t \geq 0: X(t)=x\}, \quad x>0,
$$

assuming that $c_{0}>0>c_{1}$.

We compute the conditional distributions (if the initial state is fixed) in the form

$$
\mathbb{P}_{0}\{T(x) \in \mathrm{d} t\}=\mathrm{e}^{-\lambda_{0} t} \delta_{x / c_{0}}(\mathrm{~d} t)+Q_{0}(x, t) \mathrm{d} t, \quad \mathbb{P}_{1}\{T(x) \in \mathrm{d} t\}=Q_{1}(x, t) \mathrm{d} t,
$$

where $\delta_{x / c_{0}}$ is the Dirac measure (of unit mass) at the point $x / c_{0}$ corresponding to the motion which does not change its positive initial velocity $c_{0}$. Here the $Q_{i}=Q_{i}(x, t), t \geq 0, i=0,1$, are the absolutely continuous parts of the distributions. If $t<x / c_{0}$ then the particle does not reach level $x$, and so $Q_{i}(x, t)=0$ for $i=0,1$.

We exploit again our usual notation (see (2.8) and (2.11)):

$$
\xi=\xi(x, t)=\frac{x-c_{1} t}{c_{0}-c_{1}}, \quad t-\xi=t-\xi(x, t)=\frac{c_{0} t-x}{c_{0}-c_{1}},
$$

and

$$
\theta(x, t)=\frac{1}{c_{0}-c_{1}} \exp \left\{-\lambda_{0} \xi-\lambda_{1}(t-\xi)\right\} \mathbf{1}_{\{0<\xi<t\}} .
$$

Theorem 3.1. The conditional distribution densities $Q_{0}(x, t)$ and $Q_{1}(x, t)$ can be written as follows:

$$
\begin{gathered}
Q_{0}(x, t)=\frac{\lambda_{0} \lambda_{1} x}{\sqrt{\lambda_{0} \lambda_{1} \xi(t-\xi)}} I_{1}\left(2 \sqrt{\lambda_{0} \lambda_{1} \xi(t-\xi)}\right) \theta(x, t), \\
Q_{1}(x, t)=\frac{\lambda_{1}}{\xi}\left[x I_{0}\left(2 \sqrt{\lambda_{0} \lambda_{1} \xi(t-\xi)}\right)-\frac{c_{1}}{\sqrt{\lambda_{0} \lambda_{1}}} \sqrt{\frac{t-\xi}{\xi}} I_{1}\left(2 \sqrt{\lambda_{0} \lambda_{1} \xi(t-\xi)}\right)\right] \theta(x, t) .
\end{gathered}
$$

Proof. Consider the following Laplace transform of the distribution of $T(x)$ :

$$
\phi_{i}(x, s)=\mathbb{E}_{i}\left\{\mathrm{e}^{-s T(x)}\right\}, \quad s>0, i=0,1 .
$$


In view of (3.1), the functions $\phi_{i}$ can be treated as the Laplace transform of the densities $Q_{i}$, $i=0,1$ :

$$
\phi_{0}(x, s)=\mathrm{e}^{-\left(\lambda_{0}+s\right) x / c_{0}}+\int_{x / c_{0}}^{\infty} \mathrm{e}^{-s t} Q_{0}(x, t) \mathrm{d} t, \quad \phi_{1}(x, s)=\int_{x / c_{0}}^{\infty} \mathrm{e}^{-s t} Q_{1}(x, t) \mathrm{d} t .
$$

Let $t>x / c_{0}$. Conditioning on the first velocity switching as in (2.5), we obtain the integral equations for $Q_{0}$ and $Q_{1}$.

Firstly, assuming that the first change of velocity occurs at time $\tau, \tau<x / c_{0}$, we obtain

$$
Q_{0}(x, t)=\int_{0}^{x / c_{0}} \lambda_{0} \mathrm{e}^{-\lambda_{0} \tau} Q_{1}\left(x-c_{0} \tau, t-\tau\right) \mathrm{d} \tau .
$$

Secondly, $Q_{1}(x, t)$ is separately computed under the conditions of one and more than one velocity switchings. Let $\tau$ be the first switching time. If $x-c_{1} \tau<c_{0}(t-\tau)$, i.e. if $\tau<\left(c_{0} t-x\right) / 2 c=t-\xi(x, t)$, then the particle renews its movement (at time $\tau$ from the point $\left.c_{1} \tau\right)$ with 0 -state, $\varepsilon(\tau)=0$. If $x-c_{1} \tau>c_{0}(t-\tau)$, the particle never reaches the level $x$. In the case of the strict equality $x-c_{1} \tau=c_{0}(t-\tau)$ the particle makes the only one switching at time $\tau$ and continues the movement with the velocity $c_{0}$. Therefore,

$$
\begin{aligned}
Q_{1}(x, t)= & \int_{0}^{+\infty} \lambda_{1} \mathrm{e}^{-\lambda_{1} \tau} \mathrm{e}^{-\lambda_{0}(t-\tau)} \delta\left(t-\tau-\frac{x-c_{1} \tau}{c_{0}}\right) \mathrm{d} \tau \\
& +\int_{0}^{t-\xi(x, t)} \lambda_{1} \mathrm{e}^{-\lambda_{1} \tau} Q_{0}\left(x-c_{1} \tau, t-\tau\right) \mathrm{d} \tau .
\end{aligned}
$$

Note that $t-\tau-\left(x-c_{1} \tau\right) / c_{0} \equiv\left(c_{0} t-x-2 c \tau\right) / c_{0}$ and

$$
t-\tau-\frac{x-c_{1} \tau}{c_{0}}=0 \quad \text { if and only if } \quad \tau=t-\xi(x, t)
$$

Hence,

$$
Q_{1}(x, t)=\frac{\lambda_{1} c_{0}}{2 c} \mathrm{e}^{-\lambda_{0} \xi(x, t)-\lambda_{1}(t-\xi(x, t))}+\int_{0}^{t-\xi(x, t)} \lambda_{1} \mathrm{e}^{-\lambda_{1} \tau} Q_{0}\left(x-c_{1} \tau, t-\tau\right) \mathrm{d} \tau .
$$

We obtain the following integral equations by using the Laplace transforms from (3.3), and equations (3.4)-(3.5). By applying (3.4) to the first equation of (3.3), we obtain the equation for $\phi_{0}$ :

$$
\begin{aligned}
\phi_{0}(x, s) & =\mathrm{e}^{-\left(\lambda_{0}+s\right) x / c_{0}}+\int_{x / c_{0}}^{\infty} \mathrm{e}^{-s t} \mathrm{~d} t \int_{0}^{x / c_{0}} \lambda_{0} \mathrm{e}^{-\lambda_{0} \tau} Q_{1}\left(x-c_{0} \tau, t-\tau\right) \mathrm{d} \tau \\
& =\mathrm{e}^{-\left(\lambda_{0}+s\right) x / c_{0}}+\lambda_{0} \int_{0}^{x / c_{0}} \mathrm{e}^{-\lambda_{0} \tau} \mathrm{d} \tau \int_{x / c_{0}}^{\infty} \mathrm{e}^{-s t} Q_{1}\left(x-c_{0} \tau, t-\tau\right) \mathrm{d} t \\
& =\mathrm{e}^{-\left(\lambda_{0}+s\right) x / c_{0}}+\lambda_{0} \int_{0}^{x / c_{0}} \mathrm{e}^{-\left(\lambda_{0}+s\right) \tau} \mathrm{d} \tau \int_{x / c_{0}}^{\infty} \mathrm{e}^{-s t} Q_{1}\left(x-c_{0} \tau, t\right) \mathrm{d} t .
\end{aligned}
$$

Hence, by the second equation of (3.3),

$$
\phi_{0}(x, s)=\mathrm{e}^{-\left(\lambda_{0}+s\right) x / c_{0}}+\lambda_{0} \int_{0}^{x / c_{0}} \mathrm{e}^{-\left(\lambda_{0}+s\right) \tau} \phi_{1}\left(x-c_{0} \tau, s\right) \mathrm{d} \tau .
$$


In view of (3.5), and following a calculation similar to that for (3.6), we obtain

$$
\begin{aligned}
\phi_{1}(x, s) & =\int_{x / c_{0}}^{\infty} \mathrm{e}^{-s t} Q_{1}(x, t) \mathrm{d} t \\
& =\frac{\lambda_{1} c_{0}}{2 c} \int_{x / c_{0}}^{\infty} \mathrm{e}^{-s t} \mathrm{e}^{-\lambda_{0} \xi(x, t)-\lambda_{1}(t-\xi(x, t))} \mathrm{d} t+J(x, s),
\end{aligned}
$$

where

$$
J(x, s)=\int_{x / c_{0}}^{\infty} \mathrm{e}^{-s t} \mathrm{~d} t \int_{0}^{t-\xi(x, t)} \lambda_{1} \mathrm{e}^{-\lambda_{1} \tau} Q_{0}\left(x-c_{1} \tau, t-\tau\right) \mathrm{d} \tau .
$$

By Fubini's theorem we have

$$
J(x, s)=\int_{0}^{\infty} \lambda_{1} \mathrm{e}^{-\lambda_{1} \tau-s \tau} \mathrm{d} \tau \int_{\left(x-c_{1} \tau\right) / c_{0}}^{\infty} \mathrm{e}^{-s(t-\tau)} Q_{0}\left(x-c_{1} \tau, t-\tau\right) \mathrm{d} t .
$$

Applying again the first equation of (3.3) we obtain

$$
J(x, s)=\lambda_{1} \int_{0}^{\infty} \mathrm{e}^{-\left(\lambda_{1}+s\right) \tau} \phi_{0}\left(x-c_{1} \tau, s\right) \mathrm{d} \tau-\lambda_{1} \int_{0}^{\infty} \mathrm{e}^{-\left(\lambda_{1}+s\right) \tau-\left(\lambda_{0}+s\right)\left(x-c_{1} \tau\right) / c_{0}} \mathrm{~d} \tau .
$$

We compute the latter integral substituting $\tau=\left(c_{0} t-x\right) / 2 c \equiv t-\xi(x, t)$. Note that, under this notation, $\left(x-c_{1} \tau\right) / c_{0} \equiv \xi(x, t)$. Hence,

$$
\begin{aligned}
\lambda_{1} \int_{0}^{\infty} \mathrm{e}^{-\left(\lambda_{1}+s\right) \tau-\left(\lambda_{0}+s\right)\left(x-c_{1} \tau\right) / c_{0}} \mathrm{~d} \tau & =\frac{\lambda_{1} c_{0}}{2 c} \int_{x / c_{0}}^{\infty} \mathrm{e}^{-\left(\lambda_{1}+s\right)(t-\xi(x, t))-\left(\lambda_{0}+s\right) \xi(x, t)} \mathrm{d} t \\
& =\frac{\lambda_{1} c_{0}}{2 c} \int_{x / c_{0}}^{\infty} \mathrm{e}^{-s t} \mathrm{e}^{-\lambda_{0} \xi(x, t)-\lambda_{1}(t-\xi(x, t))} \mathrm{d} t
\end{aligned}
$$

With this to hand, the equation

$$
\phi_{1}(x, s)=\lambda_{1} \int_{0}^{+\infty} \mathrm{e}^{-\left(\lambda_{1}+s\right) \tau} \phi_{0}\left(x-c_{1} \tau, s\right) \mathrm{d} \tau
$$

follows from (3.7) and (3.8).

By differentiating (3.6) and (3.9) we obtain the system of ordinary differential equations

$$
\begin{gathered}
c_{0} \frac{\partial \phi_{0}}{\partial x}(x, s)=-\left(\lambda_{0}+s\right) \phi_{0}(x, s)+\lambda_{0} \phi_{1}(x, s), \\
c_{1} \frac{\partial \phi_{1}}{\partial x}(x, s)=\lambda_{1} \phi_{0}(x, s)-\left(\lambda_{1}+s\right) \phi_{1}(x, s) .
\end{gathered}
$$

The boundary condition for $\phi_{0}$ follows from (3.6):

$$
\phi_{0}(+0, s)=1, \quad s>0 .
$$

Note that, by the definition of the first passage time, the functions $\phi_{i}, i=0,1$, vanish at $\infty$ :

$$
\phi_{0}(+\infty, s)=\phi_{1}(+\infty, s)=0, \quad s>0 .
$$

In vector form, system (3.10) reads

$$
\frac{\partial \phi}{\partial x}(x, s)=\mathcal{A} \phi(x, s), \quad x>0,
$$


where the matrix $\mathcal{A}$ is defined by

$$
\mathcal{A}=\left(\begin{array}{cc}
-\frac{\lambda_{0}+s}{c_{0}} & \frac{\lambda_{0}}{c_{0}} \\
\frac{\lambda_{1}}{c_{1}} & -\frac{\lambda_{1}+s}{c_{1}}
\end{array}\right)
$$

and $\boldsymbol{\phi}=\left(\phi_{0}, \phi_{1}\right)^{\top}$.

Equation (3.13), with boundary conditions (3.11)-(3.12), is solved in the form

$$
\phi_{0}(x, s)=\mathrm{e}^{\alpha x}, \quad \phi_{1}(x, s)=\left(1+\frac{s+\alpha c_{0}}{\lambda_{0}}\right) \mathrm{e}^{\alpha x},
$$

where $\alpha=\alpha(s)$ is the negative eigenvalue of the matrix $\mathcal{A}$, i.e. the negative root of the equation,

$$
\operatorname{det}(\mathcal{A}-\alpha I) \equiv c_{0} c_{1} \alpha^{2}+2(\tilde{a}+a s) \alpha+s(2 \lambda+s)=0 .
$$

Here we are using the notation given in (2.3) and $\tilde{a}=\left(\lambda_{1} c_{0}+\lambda_{0} c_{1}\right) / 2$. Hence,

$$
\alpha=\alpha(s)=\frac{-\tilde{a}-a s+d(s)}{c_{0} c_{1}}<0,
$$

where $d(s)=\sqrt{(\tilde{a}+a s)^{2}-c_{0} c_{1} s(2 \lambda+s)}$.

The Laplace transforms $\phi_{0}$ and $\phi_{1}$ can be inverted, with the help of [1], to yield the densities $Q_{0}$ and $Q_{1}$ in terms of modified Bessel functions.

First, we consider $Q_{0}$, defined in (3.2a):

$$
Q_{0}(x, t)=\frac{\lambda_{0} \lambda_{1} x}{\sqrt{\lambda_{0} \lambda_{1} \xi(t-\xi)}} I_{1}\left(2 \sqrt{\lambda_{0} \lambda_{1} \xi(t-\xi)}\right) \theta(x, t) .
$$

Applying the notation of (2.8) and (2.11) we obtain

$$
Q_{0}(x, t)=\frac{\sqrt{\lambda_{0} \lambda_{1}} x \exp \{-(\tilde{c} t+\beta x) / c\}}{\sqrt{\left(c_{0} t-x\right)\left(x-c_{1} t\right)}} I_{1}\left(\sqrt{\lambda_{0} \lambda_{1}\left(c_{0} t-x\right)\left(x-c_{1} t\right)} / c\right) \mathbf{1}_{\left\{t>x / c_{0}\right\}},
$$

where $\tilde{c}=\left(\lambda_{1} c_{0}-\lambda_{0} c_{1}\right) / 2$. Here the following identities have been applied:

$$
\lambda_{0} \xi+\lambda_{1}(t-\xi)=\frac{\tilde{c} t+\beta x}{c}, \quad \xi(t-\xi)=\frac{\left(c_{0} t-x\right)\left(x-c_{1} t\right)}{4 c^{2}} .
$$

Now, the Laplace transform of $Q_{0}$ can be computed by using the table; see 29.3.96 of [1]:

$$
\int_{k}^{\infty} \mathrm{e}^{-s t} \frac{a k}{\sqrt{t^{2}-k^{2}}} I_{1}\left(a \sqrt{t^{2}-k^{2}}\right) \mathrm{d} t=\mathrm{e}^{-k \sqrt{s^{2}-a^{2}}}-\mathrm{e}^{-k s} .
$$

By using the change of variables

$$
\int_{0}^{\infty} \frac{1}{c} \mathrm{e}^{(b / c) t} F\left(\frac{t}{c}\right) \mathrm{e}^{-s t} \mathrm{~d} t=\int_{0}^{\infty} \mathrm{e}^{-(c s-b) t} F(t) \mathrm{d} t
$$

we have

$$
\int_{x / c_{0}}^{\infty} \mathrm{e}^{-s t} Q_{0}(x, t) \mathrm{d} t=\mathrm{e}^{\alpha x}-\mathrm{e}^{-\left(\lambda_{0}+s\right) x / c_{0}}
$$

with $\alpha$ defined by (3.15), which is necessary to demonstrate, see (3.3) and (3.14). 
To obtain the inverse Laplace transform of $Q_{1}$, note that, with $x-c_{1} \tau$ instead of $x$ and with $t-\tau$ instead of $t,(3.16)$ becomes

$$
Q_{0}\left(x-c_{1} \tau, t-\tau\right)=\frac{\lambda_{0} \lambda_{1}\left(x-c_{1} \tau\right) I_{1}\left(2 \sqrt{\lambda_{0} \lambda_{1} \xi(t-\tau-\xi)}\right) \mathrm{e}^{-\lambda_{0} \xi-\lambda_{1}(t-\tau-\xi)}}{2 c \sqrt{\lambda_{0} \lambda_{1} \xi(t-\tau-\xi)}} .
$$

Here $\xi=\xi(x, t)=\left(x-c_{1} t\right) / 2 c$, and note that $\xi\left(x-c_{1} \tau, t-\tau\right) \equiv \xi(x, t)$. Therefore, owing to (3.5), we can write

$$
Q_{1}(x, t)=\frac{\lambda_{1} c_{0}}{2 c} \mathrm{e}^{-\lambda_{0} \xi-\lambda_{1}(t-\xi)}+\tilde{Q}_{1}(x, t)
$$

where

$$
\tilde{Q}_{1}(x, t)=\frac{\lambda_{0} \lambda_{1}^{2} \mathrm{e}^{-\lambda_{0} \xi-\lambda_{1}(t-\xi)}}{2 c} \int_{0}^{t-\xi} \frac{\left(x-c_{1} \tau\right) I_{1}\left(2 \sqrt{\lambda_{0} \lambda_{1} \xi(t-\tau-\xi)}\right)}{\sqrt{\lambda_{0} \lambda_{1} \xi(t-\tau-\xi)}} \mathrm{d} \tau .
$$

By the change the variables $z=2 \sqrt{\lambda_{0} \lambda_{1} \xi(t-\tau-\xi)}$ (or, equivalently, $\tau=\tau(z)=t-\xi-$ $\left.z^{2} /\left(4 \lambda_{0} \lambda_{1} \xi\right)\right)$, and setting $z_{0}=2 \sqrt{\lambda_{0} \lambda_{1} \xi(t-\xi)}$, we obtain

$$
\begin{aligned}
\tilde{Q}_{1}(x, t) & =\frac{\lambda_{1} \mathrm{e}^{-\lambda_{0} \xi-\lambda_{1}(t-\xi)}}{x-c_{1} t} \int_{0}^{z_{0}}\left(x-c_{1} \tau(z)\right) I_{1}(z) \mathrm{d} z \\
& =\frac{\lambda_{1} \mathrm{e}^{-\lambda_{0} \xi-\lambda_{1}(t-\xi)}}{x-c_{1} t} \int_{0}^{z_{0}}\left(c_{0} \xi(x, t)+\frac{c_{1} z^{2}}{4 \lambda_{0} \lambda_{1} \xi(x, t)}\right) I_{1}(z) \mathrm{d} z .
\end{aligned}
$$

Here we use the identity

$$
x-c_{1} \tau(z)=c_{0} \xi+\frac{c_{1} z^{2}}{4 \lambda_{0} \lambda_{1} \xi} .
$$

Then

$$
\tilde{Q}_{1}(x, t)=\frac{\lambda_{1} c_{0} \mathrm{e}^{-\lambda_{0} \xi-\lambda_{1}(t-\xi)}}{2 c} \int_{0}^{z_{0}} I_{1}(z) \mathrm{d} z+\frac{c c_{1} \mathrm{e}^{-\lambda_{0} \xi-\lambda_{1}(t-\xi)}}{2 \lambda_{0}\left(x-c_{1} t\right)^{2}} \int_{0}^{z_{0}} z^{2} I_{1}(z) \mathrm{d} z .
$$

The latter two integrals can be easily computed:

$$
\int_{0}^{z_{0}} I_{1}(z) \mathrm{d} z=I_{0}\left(z_{0}\right)-1, \quad \int_{0}^{z_{0}} z^{2} I_{1}(z) \mathrm{d} z=z_{0}^{2} I_{2}\left(z_{0}\right) ;
$$

see, e.g. 9.6.27 and 11.3.25 of [1] for $I_{0}^{\prime}(z)=I_{1}(z)$ and $\int_{0}^{z} t^{\nu} I_{\nu-1}(t) \mathrm{d} t=z^{\nu} I_{\nu}(z)$, respectively. Thus,

$$
\tilde{Q}_{1}(x, t)=\frac{\lambda_{1}}{2 c}\left[c_{0} I_{0}\left(z_{0}\right)-c_{0}+c_{1} \frac{c_{0} t-x}{x-c_{1} t} I_{2}\left(z_{0}\right)\right] \mathrm{e}^{-\lambda_{0} \xi-\lambda_{1}(t-\xi)} .
$$

The first equation in 9.6.26 of [1] is $I_{v-1}(z)-I_{v+1}(z)=(2 v / z) I_{v}(z)$, giving the identity $I_{2}\left(z_{0}\right)=I_{0}\left(z_{0}\right)-2 I_{1}\left(z_{0}\right) / z_{0}$. Hence,

$$
\tilde{Q}_{1}(x, t)=\lambda_{1}\left\{\frac{1}{x-c_{1} t}\left[x I_{0}\left(z_{0}\right)-\frac{c_{1}}{\sqrt{\lambda_{0} \lambda_{1}}} \sqrt{\frac{c_{0} t-x}{x-c_{1} t}} I_{1}\left(z_{0}\right)\right]-\frac{c_{0}}{2 c}\right\} \mathrm{e}^{-\lambda_{0} \xi-\lambda_{1}(t-\xi)} .
$$

By (3.17) and (3.18) we have

$$
Q_{1}(x, t)=\frac{\lambda_{1}}{x-c_{1} t}\left[x I_{0}\left(z_{0}\right)-\frac{c_{1}}{\sqrt{\lambda_{0} \lambda_{1}}} \sqrt{\frac{c_{0} t-x}{x-c_{1} t}} I_{1}\left(z_{0}\right)\right] \mathrm{e}^{-\lambda_{0} \xi-\lambda_{1}(t-\xi)} .
$$

Therefore, (3.2b) follows from (3.19), thus completing the proof. 
For the symmetric motion, where $c_{0}=-c_{1}=c$ and $\lambda_{0}=\lambda_{1}=\lambda,(3.2 \mathrm{a})$ and (3.2b) become

$$
\begin{gathered}
Q_{0}(x, t)=\frac{\lambda x \mathrm{e}^{-\lambda t}}{\sqrt{c^{2} t^{2}-x^{2}}} I_{1}\left(\frac{\lambda}{c} \sqrt{c^{2} t^{2}-x^{2}}\right), \\
Q_{1}(x, t)=\frac{\lambda \mathrm{e}^{-\lambda t}}{x+c t}\left[x I_{0}\left(\frac{\lambda}{c} \sqrt{c^{2} t^{2}-x^{2}}\right)+\frac{c}{\lambda} \sqrt{\frac{c t-x}{x+c t}} I_{1}\left(\frac{\lambda}{c} \sqrt{c^{2} t^{2}-x^{2}}\right)\right],
\end{gathered}
$$

which coincides with Equations (14) and (25) of [9].

Remark 3.1. For arbitrary velocity values $c_{0}, c_{1}$, with $c_{0}>0>c_{1}$, the conditional density of $T(x)$, given $\varepsilon(0)=0$, is known (see [8], [9], and [19] for symmetric motion, and [22] in the general case). In [22] an equation similar to (3.5) is derived (see (5.6) of [22]), but, to the best of our knowledge, if the initial velocity is negative (and $x>0$ ) then the explicit form of first passage time distributions $Q_{1}$ is heretofore unknown (see [22, Remark 5.1]).

For $c_{0}>c_{1}>0$, the distribution of $T(x)$ can be easily obtained. As observed in [22], the process $X=X(t), t \geq 0$, has strictly increasing sample paths and

$$
\mathbb{P}_{i}\{T(x)>t\}=\mathbb{P}_{i}\{X(t)<x\} .
$$

\section{Moments}

In this section we study the moments of $X(t), t \geq 0$. The explicit formulae for the moments can be obtained by differentiating the moment generating function $\psi_{i}(z, t)$, but it is rather cumbersome. Instead, we use an alternative approach based on the partial differential equations (2.7).

For any $t>0$, consider the moments of $X(t)$ :

$$
\mu_{n}^{(i)}(t)=\mathbb{E}_{i}\left\{X(t)^{n}\right\}=\int_{-\infty}^{\infty} x^{n} p_{i}(x, t) \mathrm{d} x, \quad i=0,1, n \geq 0 .
$$

Obviously, $\mu_{0}^{(i)}(t) \equiv 1$ for $t \geq 0$ and $i=0,1$.

Firstly, we obtain a recursive relation for $\mu_{n}(t)=\left(\mu_{n}^{(0)}(t), \mu_{n}^{(1)}(t)\right)^{\top}, n \geq 1$. We can do this using the following notation. Consider the matrices

$$
\boldsymbol{A}=\left(\begin{array}{cc}
\lambda_{1} c_{0} & \lambda_{0} c_{1} \\
\lambda_{1} c_{0} & \lambda_{0} c_{1}
\end{array}\right), \quad \boldsymbol{B}=\left(\begin{array}{cc}
\lambda_{0} c_{0} & -\lambda_{0} c_{1} \\
-\lambda_{1} c_{0} & \lambda_{1} c_{1}
\end{array}\right) .
$$

Let $\ell$ and $C$ be the integral operators defined as follows. For $f \in C[0, \infty)$,

$$
\ell f=\ell f(t):=\int_{0}^{t} f(s) \mathrm{d} s, \quad \mathcal{C} f=\mathcal{C} f(t):=\int_{0}^{t} \mathrm{e}^{-2 \lambda(t-s)} f(s) \mathrm{d} s .
$$

We use the same notation for the integral operators on $C[0, \infty) \times C[0, \infty)$ that are applied to vectors (by entries).

The following representation holds.

Theorem 4.1. For arbitrary $n \geq 1$, the moments $\mu_{n}(t)=\left(\mu_{n}^{(0)}(t), \mu_{n}^{(1)}(t)\right)^{\top}$ satisfy the recursive relation

$$
\boldsymbol{\mu}_{n}(t)=\frac{n}{2 \lambda}(\ell \boldsymbol{A}+\mathcal{C} \boldsymbol{B}) \boldsymbol{\mu}_{n-1}(t),
$$

where $2 \lambda=\lambda_{0}+\lambda_{1}$ (see the notation given in (2.3)). 
Proof. Applying the equations of (2.7), with subsequent integration by parts, to

$$
\int_{-\infty}^{\infty} x^{n} \frac{\partial p_{i}}{\partial t}(x, t) \mathrm{d} x, \quad i=0,1
$$

we obtain

$$
\frac{\mathrm{d} \mu_{n}^{(i)}(t)}{\mathrm{d} t}=n c_{i} \int_{-\infty}^{\infty} x^{n-1} p_{i}(x, t) \mathrm{d} x-\lambda_{i} \int_{-\infty}^{\infty} x^{n} p_{i}(x, t) \mathrm{d} x+\lambda_{i} \int_{-\infty}^{\infty} x^{n} p_{1-i}(x, t) \mathrm{d} x .
$$

This is equivalent to the system of ordinary differential equations

$$
\frac{\mathrm{d} \boldsymbol{\mu}_{n}(t)}{\mathrm{d} t}=\boldsymbol{\Lambda} \boldsymbol{\mu}_{n}(t)+n \tilde{\boldsymbol{\mu}}_{n-1}(t), \quad n \geq 1,
$$

with the initial conditions $\boldsymbol{\mu}_{n}(0)=\mathbf{0}, n \geq 1$, and $\boldsymbol{\mu}_{0}(t) \equiv(1,1)^{\top}$. Here $\tilde{\boldsymbol{\mu}}_{n-1}(t)=$ $\left(c_{0} \mu_{n-1}^{(0)}(t), c_{1} \mu_{n-1}^{(1)}(t)\right)^{\top}$ and the matrix $\boldsymbol{\Lambda}$ is defined by $(2.1)$.

The Cauchy problem for (4.5), with initial condition $\mathbf{0}$, is equivalent to the recursive set of the integral equations

$$
\boldsymbol{\mu}_{n}(t)=n \int_{0}^{t} \mathrm{e}^{(t-s) \Lambda} \tilde{\boldsymbol{\mu}}_{n-1}(s) \mathrm{d} s, \quad n \geq 1 .
$$

The exponential of $t \boldsymbol{\Lambda}$ can be easily calculated:

$$
\mathrm{e}^{t \boldsymbol{\Lambda}}=I-\frac{1-\mathrm{e}^{-2 \lambda t}}{2 \lambda} \boldsymbol{\Lambda}=\frac{1}{2 \lambda}\left(\begin{array}{cc}
\lambda_{1}+\lambda_{0} \mathrm{e}^{-2 \lambda t} & \lambda_{0}\left(1-\mathrm{e}^{-2 \lambda t}\right) \\
\lambda_{1}\left(1-\mathrm{e}^{-2 \lambda t}\right) & \lambda_{0}+\lambda_{1} \mathrm{e}^{-2 \lambda t}
\end{array}\right), \quad t \geq 0 .
$$

Substituting this into (4.6) we verify the integral equations (4.4), thus completing the proof.

We now derive the explicit solution of system (4.4).

Let operators $\ell$ and $\mathcal{C}$ be as defined by (4.3). Note that the product of $\ell$ and $\mathcal{C}$ is commutative. Let $U_{0,0}=U_{0,0}(t) \equiv 1$. We define the functions $U_{n, m}=U_{n, m}(t)$, where $n, m \geq 0$ and $t \geq 0$, by

$$
U_{n, m}(t):=\mathcal{C}^{n} \ell^{m} U_{0,0}(t)
$$

Note that $U_{0, m}=\ell^{m} U_{0,0}=t^{m} / m$ ! and

$$
U_{n, 0}(t)=\mathrm{e}^{n} U_{0,0}(t)=(2 \lambda)^{-n} \mathrm{e}^{-2 \lambda t} \sum_{k=n}^{\infty} \frac{(2 \lambda t)^{k}}{k !}=\frac{(2 \lambda)^{-n}}{(n-1) !} \gamma(n, 2 \lambda t),
$$

where $\gamma(n, \cdot)$ is the incomplete gamma function, $\gamma(n, x):=\int_{0}^{x} \mathrm{e}^{-t} t^{n-1} \mathrm{~d} t$ (see Formula 6.5.13 of [1]).

Applying the series expansion of the incomplete gamma function (see 6.5.29 of [1]) we obtain, for $n \geq 1$,

$$
U_{n, 0}(t)=\frac{(2 \lambda)^{-n}}{(n-1) !} \sum_{k=0}^{\infty} \frac{(-1)^{k}(2 \lambda t)^{n+k}}{k !(n+k)}=\frac{t^{n}}{(n-1) !} \sum_{k=0}^{\infty} \frac{(-2 \lambda t)^{k}}{k !(n+k)} .
$$

Furthermore, $U_{n, m}$ can be expressed by means of the confluent hypergeometric Kummer functions $\Phi=\Phi(a, b ; z)$; see (2.16). In particular, by Formula 6.5.12 of [1], we have $U_{n, 0}(t)=$ $\left(t^{n} / n !\right) \Phi(n, n+1 ;-2 \lambda t)$. Moreover, repeatedly integrating equality (4.8), we obtain, for $n, m \geq 0$,

$$
U_{n, m}(t)=\ell^{m} U_{n, 0}(t)=\frac{t^{n+m}}{(n+m) !} \Phi(n, n+m+1 ;-2 \lambda t) .
$$


It is easy to see that the functions $U_{n, m}=U_{n, m}(t), n, m \geq 0$, are linearly independent. The moments $\mu_{n}^{(i)}(t)$ can be viewed as a linear combination of $U_{n, m}$ and, consequently, of the Kummer functions; see (2.16).

Theorem 4.2. The moments $\boldsymbol{\mu}_{n}(t)$ can be expressed by means of a linear combination of the Kummer functions $\Phi(\cdot, n+1 ;-2 \lambda t)$, see (2.16), where $2 \lambda=\lambda_{0}+\lambda_{1}$ :

$$
\boldsymbol{\mu}_{n}(t)=\frac{t^{n}}{(2 \lambda)^{n}} \sum_{k=0}^{n} \boldsymbol{a}_{k, n-k} \Phi(k, n+1 ;-2 \lambda t) .
$$

Here the coefficients $\boldsymbol{a}_{n, m}=\left(a_{n, m}^{(0)}, a_{n, m}^{(1)}\right)^{\top}$ are defined by the relations

$$
\begin{gathered}
\boldsymbol{a}_{0,0}=(1,1)^{\top}, \\
\boldsymbol{a}_{0, m}=\boldsymbol{A} \boldsymbol{a}_{0, m-1}, \quad \boldsymbol{a}_{n, 0}=\boldsymbol{B} \boldsymbol{a}_{n-1,0}, \\
\boldsymbol{a}_{n, m}=\boldsymbol{A} \boldsymbol{a}_{n, m-1}+\boldsymbol{B} \boldsymbol{a}_{n-1, m},
\end{gathered}
$$

for $m \geq 1$ and $n \geq 1$, where the matrices $\boldsymbol{A}$ and $\boldsymbol{B}$ are introduced by (4.2).

Proof. Note that (4.10) is equivalent to

$$
\boldsymbol{\mu}_{n}(t)=\frac{n !}{(2 \lambda)^{n}} \sum_{k=0}^{n} \boldsymbol{a}_{k, n-k} U_{k, n-k}(t)
$$

see (4.9). Since $\mu_{0}^{(0)}=\mu_{0}^{(1)}=1$ and $U_{0,0} \equiv 1$, for $n=0$, equality (4.12) holds with $\boldsymbol{a}_{0,0}=$ $(1,1)^{\top}$.

Inserting (4.12) into the recursive integral relation (4.4) we have, for $n \geq 1$,

$$
\sum_{k=0}^{n} \boldsymbol{a}_{k, n-k} U_{k, n-k}(t)=(\boldsymbol{\ell} \boldsymbol{A}+\boldsymbol{C} \boldsymbol{B}) \sum_{k=0}^{n-1} \boldsymbol{a}_{k, n-1-k} U_{k, n-1-k}(t) .
$$

By (4.7),

$$
\ell U_{k, n-1-k}(t)=U_{k, n-k}(t) \quad \text { and } \quad \mathcal{C} U_{k, n-1-k}(t)=U_{k+1, n-1-k}(t) .
$$

Hence,

$$
\begin{aligned}
\sum_{k=0}^{n} \boldsymbol{a}_{k, n-k} U_{k, n-k}(t) & =\sum_{k=0}^{n-1} \boldsymbol{A} \boldsymbol{a}_{k, n-1-k} U_{k, n-k}(t)+\sum_{k=0}^{n-1} \boldsymbol{B} \boldsymbol{a}_{k, n-1-k} U_{k+1, n-1-k}(t) \\
& =\sum_{k=0}^{n-1} \boldsymbol{A} \boldsymbol{a}_{k, n-1-k} U_{k, n-k}(t)+\sum_{k=1}^{n} \boldsymbol{B} \boldsymbol{a}_{k-1, n-k} U_{k, n-k}(t) .
\end{aligned}
$$

Now, the equalities of (4.11) follow from the linear independence of $U_{n, m}$.

Remark 4.1. The recursive formulae (4.11) can be made closed. Note that

$$
\boldsymbol{a}_{0, m}=\boldsymbol{A}^{m} \boldsymbol{a}_{0,0}, \quad \boldsymbol{a}_{n, 0}=\boldsymbol{B}^{n} \boldsymbol{a}_{0,0}, \quad m, n \geq 1 .
$$


Set $\alpha=\operatorname{Tr}(\boldsymbol{A})=\lambda_{1} c_{0}+\lambda_{0} c_{1}$ and $\beta=\operatorname{Tr}(\boldsymbol{B})=\lambda_{0} c_{0}+\lambda_{1} c_{1}$. Since $\operatorname{det}(\boldsymbol{A})=0$ and $\operatorname{det}(\boldsymbol{B})=0$, the Cayley-Hamilton theorem yields $\boldsymbol{A}^{2}=\alpha \boldsymbol{A}$ and $\boldsymbol{B}^{2}=\beta \boldsymbol{B}$. Therefore, $\boldsymbol{A}^{m}=\alpha^{m-1} \boldsymbol{A}$ and $\boldsymbol{B}^{n}=\beta^{n-1} \boldsymbol{B}$ for $m, n \geq 1$. Thus,

$$
a_{0, m}^{(0)}=a_{0, m}^{(1)}=\left(\lambda_{1} c_{0}+\lambda_{0} c_{1}\right)^{m}, \quad m \geq 0,
$$

and

$$
\begin{gathered}
a_{n, 0}^{(0)}=\lambda_{0}\left(c_{0}-c_{1}\right)\left(\lambda_{0} c_{0}+\lambda_{1} c_{1}\right)^{n-1}, \\
a_{n, 0}^{(1)}=-\lambda_{1}\left(c_{0}-c_{1}\right)\left(\lambda_{0} c_{0}+\lambda_{1} c_{1}\right)^{n-1}, \quad n \geq 1 .
\end{gathered}
$$

After some cumbersome algebra we obtain the closed expressions for $\boldsymbol{a}_{n, m}$, with arbitrary $n \geq 1$ and $m \geq 0$ :

$$
a_{n, m}^{(0)}=d_{n, m}-2 \lambda c_{1} d_{n-1, m}, \quad a_{n, m}^{(1)}=d_{n, m}-2 \lambda c_{0} d_{n-1, m} .
$$

Here

$$
d_{n, m}=\sum_{k: \max \{n, m\} \leq k \leq n+m} \frac{\alpha^{k-n} \beta^{k-m}(-\gamma)^{n+m-k} k !}{(k-n) !(k-m) !(n+m-k) !},
$$

with $\gamma=4 c_{0} c_{1} \lambda^{2}$. This gives us the closed formulae for the moments; see (4.10).

Remark 4.2. For the symmetric telegraph process, (4.10) becomes more simple. If we have $\lambda_{0}=\lambda_{1}=: \lambda$ and $c_{0}=-c_{1}=: c$ then $\alpha=\operatorname{Tr}(\boldsymbol{A})=0, \beta=\operatorname{Tr}(\boldsymbol{B})=0$, and $\gamma=-4 c^{2} \lambda^{2}$. Owing to (4.16) we obtain $d_{n, m}=0$, if $n \neq m$, and $d_{n, n}=\left(4 c^{2} \lambda^{2}\right)^{n}$. Hence, (4.10) can be easily simplified to

$$
\begin{gathered}
\mu_{2 n}^{(i)}(t)=(c t)^{2 n} \Phi(n, 2 n+1 ;-2 \lambda t), \\
\mu_{2 n+1}^{(i)}(t)=(-1)^{i}(c t)^{2 n+1} \Phi(n+1,2 n+2 ;-2 \lambda t) .
\end{gathered}
$$

Formulae for the moments of the symmetric telegraph processes are presented in [12] and [14] in terms of modified Bessel functions, but they are not as concise as (4.17).

For $|z| \rightarrow \infty, \operatorname{Re} z<0$, the Kummer function has the following asymptotics (see 13.1.5 of [1]):

$$
\Phi(a, b ; z)=\frac{\Gamma(b)}{\Gamma(b-a)}(-z)^{-a}\left[1+O\left(|z|^{-1}\right)\right] .
$$

Applying (4.18) to (4.17) we obtain the limits under Kac's rescaling: if $c$ and $\lambda \rightarrow \infty$ are such that $c^{2} / \lambda \rightarrow \sigma^{2}$, then

$$
\mu_{2 n}^{(i)}(t) \rightarrow\left(\sigma^{2} t\right)^{n}(2 n-1) ! !, \quad \mu_{2 n+1}^{(i)}(t) \rightarrow 0, \quad i=0,1 .
$$

These limits coincide with moments of Brownian motion with diffusion coefficient $\sigma^{2}$. It corresponds to the classical result by Kac [13].

\section{Kac's scaling}

In this section we generalise to the asymmetrical situation the limits obtained in (4.19) for the symmetric case. Assume that the switching intensities are large, but comparable, i.e. $\lambda_{0}, \lambda_{1} \rightarrow$ $\infty$ and

$$
\frac{\lambda_{0}}{\lambda_{1}} \rightarrow v^{2}, \quad v \in(0, \infty)
$$


The Kac condition is assumed for both of the states separately, i.e. $c_{0} \rightarrow+\infty, c_{1} \rightarrow-\infty$, and $\lambda_{0}, \lambda_{1} \rightarrow+\infty$ such that

$$
\frac{c_{0}}{\sqrt{\lambda_{0}}} \rightarrow \sigma_{0}, \quad \frac{c_{1}}{\sqrt{\lambda_{1}}} \rightarrow-\sigma_{1}
$$

where $\sigma_{0}, \sigma_{1}>0$. From (5.1)-(5.2), it follows that the velocities are comparable as well:

$$
\frac{c_{0}}{c_{1}}=\frac{c_{0} / \sqrt{\lambda_{0}}}{c_{1} / \sqrt{\lambda_{1}}} \sqrt{\lambda_{0} / \lambda_{1}} \rightarrow-\frac{v \sigma_{0}}{\sigma_{1}} .
$$

We also assume that

$$
\frac{\lambda_{1} c_{0}+\lambda_{0} c_{1}}{\lambda_{1}+\lambda_{0}} \rightarrow \delta
$$

The latter limit relation is equivalent to

$$
c_{1} \frac{c_{0} / c_{1}+\lambda_{0} / \lambda_{1}}{1+\lambda_{0} / \lambda_{1}} \rightarrow \delta .
$$

Hence, $c_{0} / c_{1}+\lambda_{0} / \lambda_{1} \rightarrow 0$ and $v=\sigma_{0} / \sigma_{1}$. So, condition (5.3) reads as the rate of 'similarity' between $\lambda_{0} / \lambda_{1}$ and $c_{0} / c_{1}$ at $\infty$. More precisely,

$$
c_{1}\left(\frac{c_{0}}{c_{1}}+\frac{\lambda_{0}}{\lambda_{1}}\right) \rightarrow \delta\left(1+v^{2}\right) .
$$

Note that (5.1) and (5.2) have the following consequences:

$$
\begin{gathered}
\frac{c_{0}^{2}}{\lambda_{0}+\lambda_{1}} \rightarrow \frac{v^{2} \sigma_{0}^{2}}{1+v^{2}}=\frac{\sigma_{0}^{4}}{\sigma_{0}^{2}+\sigma_{1}^{2}}, \quad \frac{c_{1}^{2}}{\lambda_{0}+\lambda_{1}} \rightarrow \frac{\sigma_{1}^{2}}{1+v^{2}}=\frac{\sigma_{1}^{4}}{\sigma_{0}^{2}+\sigma_{1}^{2}}, \\
\frac{c_{0} c_{1}}{\lambda_{0}+\lambda_{1}} \rightarrow-\frac{v \sigma_{0} \sigma_{1}}{1+v^{2}}=-\frac{\sigma_{0}^{2} \sigma_{1}^{2}}{\sigma_{0}^{2}+\sigma_{1}^{2}} .
\end{gathered}
$$

Therefore,

$$
\frac{\left(c_{0}-c_{1}\right)^{2}}{\lambda_{0}+\lambda_{1}} \rightarrow \sigma_{0}^{2}+\sigma_{1}^{2} \quad \text { and } \quad \frac{\left(c_{0}+c_{1}\right)^{2}}{\lambda_{0}+\lambda_{1}} \rightarrow \frac{\left(\sigma_{0}^{2}-\sigma_{1}^{2}\right)^{2}}{\sigma_{0}^{2}+\sigma_{1}^{2}}
$$

Note that if $v \neq 1$ then the scaling conditions (5.1)-(5.3) lead to $c_{0}+c_{1} \rightarrow \infty$ (see (5.5)). Certainly, $c_{0}-c_{1} \rightarrow \infty$.

Theorem 5.1. Under the scaling conditions (5.1)-(5.3), the moments $\mu_{n}^{(i)}(t)$ defined by (4.1) converge as

$$
\mu_{n}^{(i)}(t) \rightarrow n ! \sum_{k=0}^{[n / 2]} \frac{\delta^{n-2 k} \kappa^{2 k} t^{n-k}}{k !(n-2 k) !}
$$

where $\kappa^{2}=\sigma_{0}^{2} \sigma_{1}^{2} /\left(\sigma_{0}^{2}+\sigma_{1}^{2}\right)$, and $[n / 2]$ denotes the integer part of the number $n / 2$.

Proof. We begin with the limits

$$
A_{k, n}^{(i)}=\lim \frac{a_{k, n-k}^{(i)}}{(2 \lambda)^{n+k}}, \quad 0 \leq k \leq n,
$$

where $2 \lambda=\lambda_{0}+\lambda_{1}$. Here lim denotes the limit under the scaling conditions (5.1)-(5.3), and $a_{k, n-k}^{(i)}$ are defined by (4.11); see (4.13)-(4.15) as well. 
First, on the boundary, i.e. for $k=0$ and $k=n$, owing to (4.13) and (4.14) the limits $A_{0, n}^{(i)}$ and $A_{n, n}^{(i)}$ are given by

$$
A_{0, n}^{(i)}=\lim a_{0, n}^{(i)}=\lim \left(\frac{\lambda_{1} c_{0}+\lambda_{0} c_{1}}{2 \lambda}\right)^{n}=\delta^{n}, \quad n \geq 0, i=0,1,
$$

and

$$
\begin{aligned}
A_{n, n}^{(i)} & =\lim \frac{a_{n, 0}^{(i)}}{(2 \lambda)^{2 n}} \\
& =(-1)^{i} \lim \lambda_{i}\left(c_{0}-c_{1}\right) \frac{\left(\lambda_{0} c_{0}+\lambda_{1} c_{1}\right)^{n-1}}{(2 \lambda)^{2 n}} \\
& =(-1)^{i} \lim \frac{\lambda_{i}}{2 \lambda} \lim \frac{c_{0}-c_{1}}{2 \lambda} \lim \frac{\left(\lambda_{0} c_{0}+\lambda_{1} c_{1}\right)^{n-1}}{(2 \lambda)^{2 n-2}} \\
& =0, \quad n \geq 1, i=0,1 .
\end{aligned}
$$

We applied here (5.3) for (5.7), and (5.1), (5.3), and (5.5) for (5.8).

In general, we prove that the limits $A_{k, n}^{(i)}=A_{k, n}, 0 \leq k \leq n$, are independent of $i$ and

$$
\begin{gathered}
A_{k, n}=\left(\begin{array}{c}
n-k \\
k
\end{array}\right) \delta^{n-2 k} \kappa^{2 k} \quad \text { if } k \leq[n / 2], \\
A_{k, n}=0 \quad \text { if } k>[n / 2],
\end{gathered}
$$

where (5.9) uses the binomial coefficient. We proceed by induction on $n$.

For $n=0$ and $n=1,(5.9)-(5.10)$ are given by (5.7) and (5.8).

Let us begin the proof with $n=2$. The limits $A_{0,2}=\delta^{2}$ and $A_{2,2}=0$ are given by (5.7) and (5.8). For $A_{1,2}^{(i)}=\lim \left(a_{1,1}^{(i)} /(2 \lambda)^{3}\right)$, note that (4.15) and (4.16) give

$$
\begin{aligned}
\frac{a_{1,1}^{(i)}}{(2 \lambda)^{3}} & =\frac{d_{11}}{(2 \lambda)^{3}}-\frac{c_{1-i} d_{01}}{(2 \lambda)^{2}} \\
& =-\frac{c_{0} c_{1}}{2 \lambda}+\frac{\left(\lambda_{1} c_{0}+\lambda_{0} c_{1}\right)\left(\lambda_{0} c_{0}+\lambda_{1} c_{1}\right)}{4 \lambda^{3}}-\frac{c_{1-i}\left(\lambda_{1} c_{0}+\lambda_{0} c_{1}\right)}{4 \lambda^{2}} .
\end{aligned}
$$

Owing to (5.4), the first term converges to $\kappa^{2}$ and the rest vanish by (5.3) and (5.2). Thus, $A_{1,2}^{(i)}=\kappa^{2}$, which is in accordance with (5.9).

We continue by induction. Owing to (4.11), for any $n \geq 3$ and $0<k<n$, we have the identities

$$
\frac{a_{k, n-k}^{(i)}}{(2 \lambda)^{n+k}}=\frac{\lambda_{1} c_{0} a_{k, n-1-k}^{(0)}+\lambda_{0} c_{1} a_{k, n-1-k}^{(1)}}{(2 \lambda)^{n+k}}+(-1)^{i} \frac{\lambda_{i}\left(c_{0} a_{k-1, n-k}^{(0)}-c_{1} a_{k-1, n-k}^{(1)}\right)}{(2 \lambda)^{n+k}}
$$

for $i=0,1$. The latter term vanishes, i.e.

$$
\frac{\lambda_{i}\left(c_{0} a_{k-1, n-k}^{(0)}-c_{1} a_{k-1, n-k}^{(1)}\right)}{(2 \lambda)^{n+k}}=\frac{\lambda_{i}}{2 \lambda}\left[\frac{c_{0}}{2 \lambda} \frac{a_{k-1, n-k}^{(0)}}{(2 \lambda)^{n+k-2}}-\frac{c_{1}}{2 \lambda} \frac{a_{k-1, n-k}^{(1)}}{(2 \lambda)^{n+k-2}}\right] \rightarrow 0,
$$

since the limits

$$
\lim \frac{\lambda_{i}}{2 \lambda}, \quad \lim \frac{a_{k-1, n-k}^{(i)}}{(2 \lambda)^{n+k-2}}, \quad i=0,1,
$$


are finite (the second limit is finite by the induction hypotheses) and, by (5.2), $\lim \left(c_{i} / 2 \lambda\right)=0$. Therefore, the limit of $a_{k, n-k}^{(i)} /(2 \lambda)^{n+k}$ is independent of $i$. Furthermore, we have

$$
\lim \frac{a_{k, n-k}^{(i)}}{(2 \lambda)^{n+k}}=\lim \frac{a_{k, n-1-k}^{(1)}\left(\lambda_{1} c_{0}+\lambda_{0} c_{1}\right)}{(2 \lambda)^{n+k}}+\lim \frac{\lambda_{1} c_{0}\left(a_{k, n-1-k}^{(0)}-a_{k, n-1-k}^{(1)}\right)}{(2 \lambda)^{n+k}} .
$$

By (4.15) (or directly by (4.11)) we obtain

$$
a_{k, n-1-k}^{(0)}-a_{k, n-1-k}^{(1)}=2 \lambda\left(c_{0} a_{k-1, n-1-k}^{(0)}-c_{1} a_{k-1, n-1-k}^{(1)}\right) .
$$

Hence (see (5.4)),

$$
\begin{aligned}
\lim & \frac{\lambda_{1} c_{0}\left(a_{k, n-1-k}^{(0)}-a_{k, n-1-k}^{(1)}\right)}{(2 \lambda)^{n+k}} \\
& =\lim \frac{\lambda_{1} c_{0}\left(c_{0} a_{k-1, n-1-k}^{(0)}-c_{1} a_{k-1, n-1-k}^{(1)}\right)}{(2 \lambda)^{n+k-1}} \\
& =\lim \frac{\lambda_{1}}{2 \lambda}\left[\lim \frac{c_{0}^{2}}{2 \lambda}-\lim \frac{c_{0} c_{1}}{2 \lambda}\right] A_{k-1, n-2} \\
& =\frac{\sigma_{1}^{2}}{\sigma_{0}^{2}+\sigma_{1}^{2}}\left[\frac{\sigma_{0}^{4}}{\sigma_{0}^{2}+\sigma_{1}^{2}}+\frac{\sigma_{0}^{2} \sigma_{1}^{2}}{\sigma_{0}^{2}+\sigma_{1}^{2}}\right] A_{k-1, n-2} \\
& =\frac{\sigma_{0}^{2} \sigma_{1}^{2}}{\sigma_{0}^{2}+\sigma_{1}^{2}} A_{k-1, n-2} .
\end{aligned}
$$

Finally, passing to the limit in (5.11), we obtain the following equation:

$$
A_{k, n}=\delta A_{k, n-1}+\kappa^{2} A_{k-1, n-2} .
$$

By induction using the 'boundary conditions' (5.7)-(5.8) we prove that the recursive equation (5.12) has the (unique) solution defined by (5.9)-(5.10).

To complete the proof of Theorem 5.1, we apply the asymptotics (4.18) of the Kummer function and the limits calculated by (5.9)-(5.10) to (4.10):

$$
\begin{aligned}
\mu_{n}^{(i)}(t) & =\frac{t^{n}}{(2 \lambda)^{n}} \sum_{k=0}^{n} a_{k, n-k}^{(i)} \Phi(k, n+1 ;-2 \lambda t) \\
& \sim \frac{t^{n}}{(2 \lambda)^{n}} \sum_{k=0}^{n} \frac{a_{k, n-k}^{(i)}}{(2 \lambda t)^{k}} \frac{\Gamma(n+1)}{\Gamma(n-k+1)} \\
& \rightarrow n ! \sum_{k=0}^{n} \frac{A_{k, n} t^{n-k}}{(n-k) !} \\
& =n ! \sum_{k=0}^{[n / 2]}\left(\begin{array}{c}
n-k \\
k
\end{array}\right) \delta^{n-2 k} \kappa^{2 k} \frac{t^{n-k}}{(n-k) !} \\
& =n ! \sum_{k=0}^{[n / 2]} \frac{\delta^{n-2 k} \kappa^{2 k} t^{n-k}}{k !(n-2 k) !} .
\end{aligned}
$$


Corollary 5.1. Under the scaling conditions (5.1)-(5.3), the marginal distributions of the telegraph process converge to the distributions of a diffusion:

$$
X(t) \stackrel{\mathrm{D}}{\rightarrow} \sigma w_{t}+\delta t, \quad t>0 .
$$

Here $w_{t} \sim \mathcal{N}(0, t)$ and

$$
\sigma=\kappa \sqrt{2}=\frac{\sigma_{0} \sigma_{1}}{\sqrt{\left(\sigma_{0}^{2}+\sigma_{1}^{2}\right) / 2}} .
$$

Proof. As is easy to see, the limits in (5.6) coincide with the moments of the diffusion $\sigma w_{t}+\delta t, t \geq 0$, with $\sigma=\kappa \sqrt{2}$. The convergence in distribution (5.13) follows from [3, Theorem 30.2].

\section{Acknowledgements}

This research has been partly supported by the Research Fund of Rosario University (FIUR), project DVG140. We are grateful to the anonymous referee for carefully reading this paper and for his/her useful comments.

\section{References}

[1] Abramowitz, M. And Stegun, I. A. (eds) (1965). Handbook of Mathematical Functions, with Formulas, Graphs, and Mathematical Tables. Dover, New York.

[2] Beghin, L., Nieddu, L. And Orsingher, E. (2001). Probabilistic analysis of the telegrapher's process with drift by means of relativistic transformations. J. Appl. Math. Stoch. Anal. 14, 11-25.

[3] Billingsley, P. (1986). Probability and Measure, 2nd edn. John Wiley, New York.

[4] Bogachev, L. and Ratanov, N. (2011). Occupation time distributions for the telegraph process. Stoch. Process. Appl. 121, 1816-1844.

[5] Bshouty, D., Di Crescenzo, A., Martinucci, B. And Zacks, S. (2012). Generalized telegraph process with random delays. J. Appl. Prob. 49, 850-865.

[6] De Gregorio, A., Orsingher, E. And Sakhno, L. (2005). Motions with finite velocity analyzed with order statistics and differential equations. Theory Prob. Math. Statist. 71, 63-79.

[7] Di Crescenzo, A. And Martinucci, B. (2013). On the generalized telegraph process with deterministic jumps. Methodology Comput. Appl. Prob. 15, 215-235.

[8] Foong, S. K. (1992). First-passage time, maximum displacement, and Kac's solution of the telegrapher equation. Phys. Rev. A. 46, 707-710.

[9] Foong, S. K. And Kanno, S. (1994). Properties of the telegrapher's random process with or without a trap. Stoch. Process. Appl. 53, 147-173.

[10] Hadeler, K. P. (1999). Reaction transport systems in biological modelling. In Mathematics Inspired by Biology (Lecture Notes in Math. Vol. 1714), eds V. Capasso and O. Diekmann, Springer, Berlin, pp. 95-150.

[11] Hillen, T. and Hadeler, K. P. (2005). Hyperbolic systems and transport equations in mathematical biology. In Analysis and Numerics for Conservation Laws, ed. G. Warnecke, Springer, Berlin, pp. 257-279.

[12] Iacus, S. ANd Yoshida, N. (2009). Estimation for the discretely observed telegraph process. Theory Prob. Math. Statist. 78, 37-47.

[13] KAC, M. (1974). A stochastic model related to the telegrapher's equation. Rocky Mountain J. Math. 4, 497-509.

[14] Kolesnik, A. D. (2012). Moment analysis of the telegraph random process. Bull. Acad. Sci. Moldova, Ser. Math. 68, 90-107.

[15] López, O. and Ratanov, N. (2012). Kac's rescaling for jump-telegraph processes. Statist. Prob. Lett. 82, 1768-1776.

[16] Окиво, A. And Levin, S. A. (2001). Diffusion and Ecological Problems: Modern Perspectives, 2nd edn. (Interdisciplinary Appl. Math. 14). Springer, New York.

[17] Orsingher, E. (1990). Probability law, flow function, maximum distribution of wave-governed random motions and their connections with Kirchhoff's laws. Stoch. Process. Appl. 34, 49-66.

[18] ORsingher, E. (1995). Motions with reflecting and absorbing barriers driven by the telegraph equation. Random Operators Stoch. Equat. 3, 9-21.

[19] Pinsky, M. A. (1991). Lectures on Random Evolution. World Scientific Publishing Co. River Edge, New Jersey. 
[20] Ratanov, N. (1999). Telegraph evolutions in inhomogeneous media. Markov Process. Relat. Fields 5, 53-68.

[21] Ratanov, N. (2007). A jump telegraph model for option pricing. Quant. Finance 7, 575-583.

[22] Stadje, W. And Zacks, S. (2004). Telegraph processes with random velocities. J. Appl. Prob. 41, $665-678$.

[23] Weiss, G. H. (2002). Some applications of persistent random walks and the telegrapher's equation. Physica A. 311, 381-410.

[24] ZACKS, S. (2004). Generalized integrated telegraph processes and the distribution of related stopping times. J. Appl. Prob. 41, 497-507. 